\title{
Linking Void and Interphase Evolution to Electrochemistry in Solid-State Batteries Using Operando X-Ray Tomography
}

John A. Lewis ${ }^{1}$, Francisco Javier Quintero Cortes ${ }^{1}$, Yuhgene Liu ${ }^{1}$, John C. Miers ${ }^{2}$, Ankit Verma ${ }^{3}$, Bairav S. Vishnugopi ${ }^{3}$, Jared Tippens ${ }^{2}$, Dhruv Prakash ${ }^{1}$, Thomas S. Marchese ${ }^{1}$, Sang Yun Han ${ }^{2}$, Chanhee Lee ${ }^{2,4}$, Hyun-Wook Lee ${ }^{4}$, Pavel Shevchenko, Francesco De Carlo ${ }^{5}$, Christopher Saldana $^{2}$, Partha P. Mukherjee ${ }^{3}$, Matthew T. McDowell ${ }^{1,2 *}$

${ }^{1}$ School of Materials Science and Engineering, Georgia Institute of Technology, Atlanta, GA, USA ${ }^{2}$ George W. Woodruff School of Mechanical Engineering, Georgia Institute of Technology, Atlanta, GA, USA

${ }^{3}$ School of Mechanical Engineering, Purdue University, West Lafayette, IN, USA

${ }^{4}$ School of Energy and Chemical Engineering, Ulsan National Institute of Science and Technology, Ulsan, Republic of Korea

${ }^{5}$ Advanced Photon Source, Argonne National Laboratory, Lemont, IL, USA

*Corresponding Author: mattmcdowell@gatech.edu 


\begin{abstract}
Despite progress in solid-state battery engineering, our understanding of the chemo-mechanical phenomena that govern electrochemical behavior and stability at solid-solid interfaces remains limited compared to solid-liquid interfaces. Here, we use operando synchrotron X-ray computed microtomography to investigate the evolution of lithium/solid-state electrolyte interfaces during battery cycling, revealing how the complex interplay between void formation, interphase growth, and volumetric changes determines cell behavior. Void formation during lithium stripping is directly visualized in symmetric cells, and the loss of contact at the interface between lithium and the solid-state electrolyte $\left(\mathrm{Li}_{10} \mathrm{SnP}_{2} \mathrm{~S}_{12}\right)$ is found to be the primary cause of cell failure. Reductive interphase formation within the solid-state electrolyte is simultaneously observed, and image segmentation reveals that the interphase is redox-active upon charge. At the cell level, we postulate that global volume changes and loss of stack pressure occur due to partial molar volume mismatches at either electrode. These results provide new insight into how chemo-mechanical phenomena can impact cell performance, which is necessary to understand for the development of solid-state batteries.
\end{abstract}




\section{$\underline{\text { Introduction }}$}

Solid-state batteries (SSBs) have garnered interest due to their lack of hazardous liquid electrolytes, as well as the potential of solid-state electrolytes (SSEs) to suppress lithium filament growth and thereby enable lithium metal anodes ${ }^{1-4}$. Despite advances in the development of SSEs with improved ionic conductivity ${ }^{5-11}$, the understanding and control over solid electrode/SSE interfaces have emerged as major challenges in the development of $\mathrm{SSBs}^{2,3,12-14}$. In general, chemo-mechanical degradation is expected to be more severe in SSBs compared to conventional liquid-electrolyte-batteries because the SSE cannot reconfigure and flow like liquids ${ }^{2,12}$. Understanding chemo-mechanical phenomena, such as chemical transformations at interfaces, mechanical damage, and lithium filament growth, is therefore critical for engineering SSBs.

Most SSEs are electrochemically unstable against lithium metal and decompose to form an interphase layer at the interface ${ }^{15-17}$, with the transport properties and structure of the interphase playing a crucial role in determining material evolution and degradation pathways ${ }^{18-24}$. Additionally, SSEs that form passivating interphases (such as $\mathrm{Li}_{7} \mathrm{La}_{3} \mathrm{Zr}_{2} \mathrm{O}_{12}$ and the $\mathrm{Li}_{2} \mathrm{~S}_{-} \mathrm{P}_{2} \mathrm{~S}_{5}$ system) are often vulnerable to lithium metal penetration that results in short circuits ${ }^{25-28}$. This phenomenon limits these cells to a critical current density that is typically below $1 \mathrm{~mA} \mathrm{~cm}^{-2}$, insufficient for commercial batteries ${ }^{29}$. Furthermore, establishing and maintaining mechanical contact at Li/SSE interfaces during cycling is challenging. Morphological and volumetric changes of electrode materials and interphases can result in contact loss or other mechanical damage ${ }^{20,30-}$ 33. Although engineering strategies have been implemented to improve interfacial contact ${ }^{34-37}$, electrochemical studies using three-electrode cells have shown that the operation of Li/SSE interfaces at high rates is fundamentally limited by the formation of voids during lithium stripping $^{38-40}$. Void formation is detrimental to cell performance, as the reduced contact area 
creates an effectively larger current density and hot spots that can drive lithium metal penetration $^{38,39}$.

While a variety of studies have provided evidence for these phenomena, there is a need for operando experiments to directly elucidate how solid-state interfaces evolve in real time, and how the interplay among various chemo-mechanical phenomena impacts electrochemical behavior. However, probing buried interfaces in SSBs is challenging, since it requires techniques that can penetrate deep within materials while providing local information about interfacial regions. X-ray tomography is an excellent characterization tool for studying such electrochemical systems, as it yields three-dimensional images of materials with spatial resolution down to the sub-micron scale $^{41,42}$. X-ray tomography has recently been used in a variety of SSB studies ${ }^{31,33,38,43-49}$, revealing information about how cell performance is impacted by factors such as structure, porosity, and mechanical properties of materials. However, most X-ray tomography experiments investigating $\mathrm{Li} / \mathrm{SSE}$ interfaces have been limited to ex situ studies or long scan times, making it difficult to resolve meaningful dynamics at these interfaces during electrochemical cycling.

In this work, we used operando synchrotron X-ray computed microtomography to observe dynamic phenomena during operation of $\mathrm{Li} / \mathrm{Li}_{10} \mathrm{SnP}_{2} \mathrm{~S}_{12} / \mathrm{Li}$ symmetric cells at the relatively high nominal current densities of $1 \mathrm{~mA} \mathrm{~cm}^{-2}$ and $4 \mathrm{~mA} \mathrm{~cm}^{-2}$. Taking advantage of the high spatial resolution and fast scan times possible with the use of a monochromatic synchrotron beam, we were able to simultaneously resolve void formation and interphase evolution at the $\mathrm{Li} / \mathrm{L}_{10} \mathrm{SnP}_{2} \mathrm{~S}_{12}$ interface during cycling. We found that interphase growth is severe due to the mixed-conducting properties of the decomposed phase, and that interphase formation is favored over plating lithium metal. Although this decomposition is irreversible, we observed that the interphase is redox active upon charge. Additionally, volumetric changes within the cell were found to be due to mismatches 
in partial molar volume at each electrode, resulting in nonuniform strain states across the cell. Despite the substantial phase transformations and volume changes at the interfaces, our analysis and modeling showed that cell failure was ultimately driven by void formation and interfacial contact loss. These results improve our understanding of chemo-mechanics in the context of void formation, interphase growth, and volumetric changes at solid-state interfaces, which are processes that must be controlled for the development of SSBs.

\section{Operando X-Ray Tomography Experiments}

The SSE studied in this work was the sulfide $\mathrm{Li}_{10} \mathrm{SnP}_{2} \mathrm{~S}_{12}$ (LSPS). The crystal structure of pristine LSPS was verified using X-ray diffraction (Fig. S1) ${ }^{50}$. The conductivity of the as-received LSPS was measured to be $2.1 \mathrm{mS} \mathrm{cm}^{-1}$ at $20^{\circ} \mathrm{C}$ (Fig. S2), which is high enough to cycle at current densities comparable to the targeted level for commercial cells ${ }^{29}$. However, the Li/LSPS interface is unstable and will decompose through (electro)chemical reactions ${ }^{52,53}$. Since the interphase formed between lithium and LSPS has mixed-conducting properties, this reaction is not selfpassivating during cycling and decomposition occurs readily ${ }^{17}$. Although these interfacial reactions are undesirable, the properties of LSPS provide a route to understanding the complex coupled dynamics that occur at Li/SSE interfaces when cycled at realistic current densities, including the impact of interphase formation on cell capacity, stability, and redox processes.

Operando X-ray computed microtomography experiments were conducted at beamline 2BM at the Advanced Photon Source. A custom solid-state cell was designed to apply a stack pressure to a $2 \mathrm{~mm}$-diameter solid-state cell while maintaining an airtight seal, as shown in Fig. 1a. Scaling the internal cell diameter down to $2 \mathrm{~mm}$ (compared to $\sim 10 \mathrm{~mm}$ for conventional research-grade SSB cells ${ }^{48,51}$ ) was critical to achieve sufficient X-ray transmission through LSPS. Monochromatic X-rays with an energy of $28 \mathrm{keV}$ were used, as this energy provided greater 
transmission than higher-energy X-rays because of an absorption edge of LSPS at $29.2 \mathrm{keV}$ (Fig.

S3). We were able to position the entire sample within the field of view to obtain a voxel size of

$1.7 \mu \mathrm{m}$ while using a rotational tomographic scan time of approximately 7 min. Full details are presented in the Methods section.

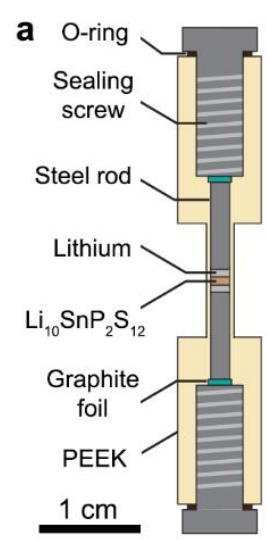

b
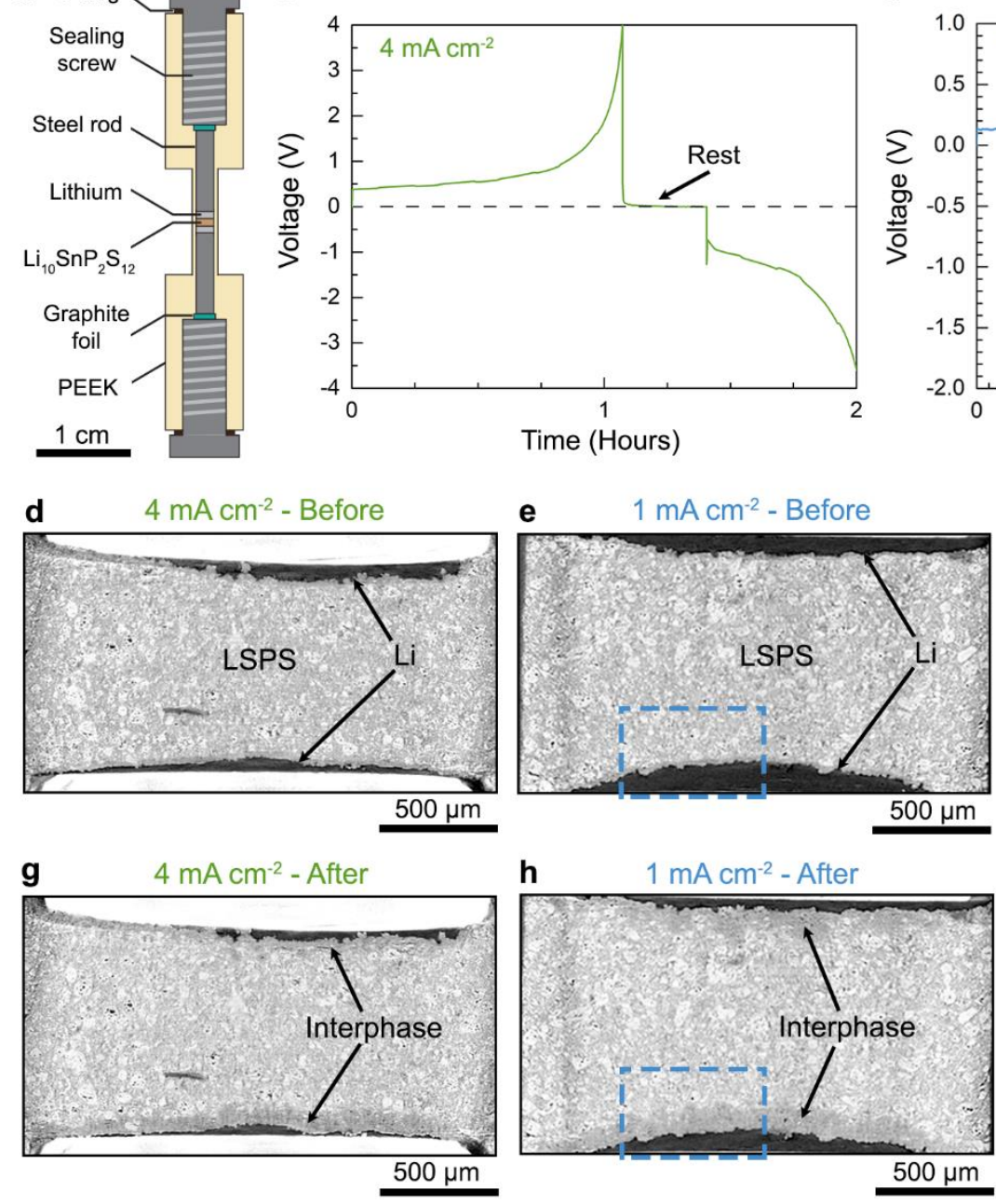
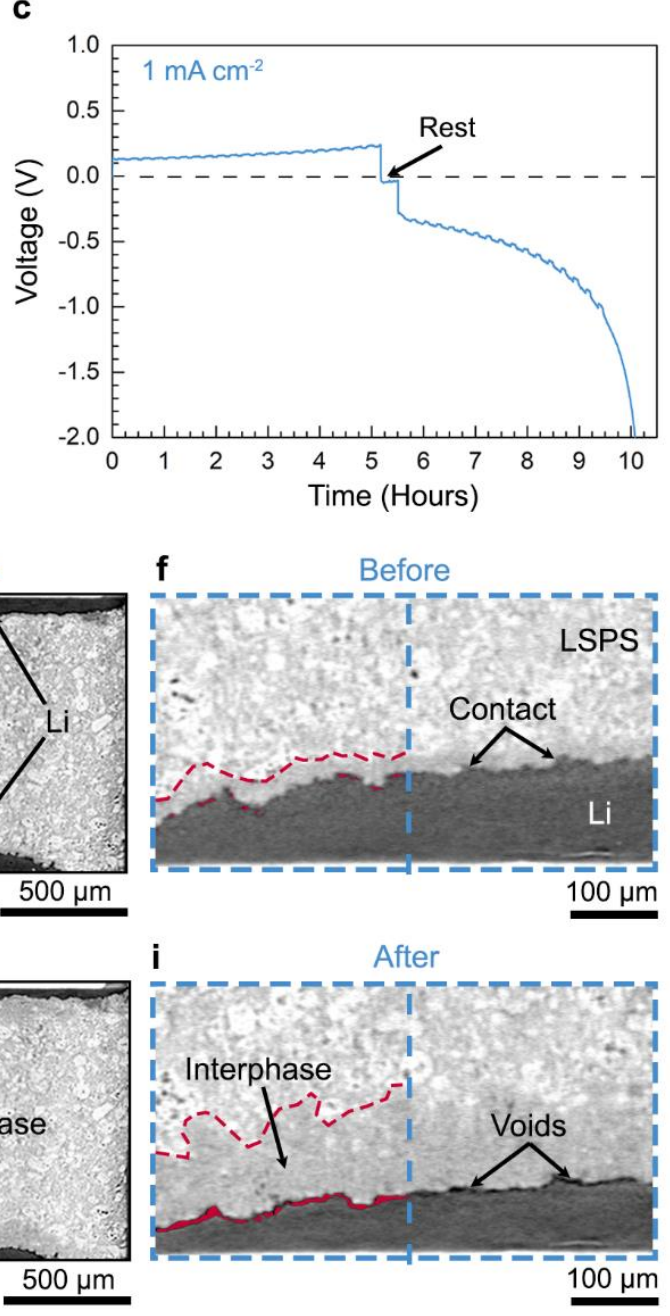

Figure 1. Operando X-ray imaging of cells at two current densities. (a) Schematic of the custom $\mathrm{X}$-ray tomography cell used to cycle $\mathrm{Li} / \mathrm{LSPS} / \mathrm{Li}$ cells during operando experiments. (b-c) Galvanostatic voltage curves measured during operando experiments at $4 \mathrm{~mA} \mathrm{~cm}^{-2}$ and $1 \mathrm{~mA} \mathrm{~cm}^{-}$ ${ }^{2}$, respectively. (d-e) Reconstructed cross-sectional images before cycling at $4 \mathrm{~mA} \mathrm{~cm}{ }^{-2}$ and $1 \mathrm{~mA}$ $\mathrm{cm}^{-2}$. The regions with dark contrast are the lithium electrodes, while the grey phase is the LSPS electrolyte. (f) Magnified cross-section of the Li/LSPS interface before cycling at $1 \mathrm{~mA} \mathrm{~cm}^{-2}$, taken from the blue-boxed region in (e). Voids in the left half of the image are overlaid with red for easier visualization, and the red dashed line on the left side demarcates the interphase boundary. The right half of the image is unmarked. (g-h) The same cross-sectional images as those shown in (d-e) after the electrochemical cycling procedure shown in (b-c). The formation of a darker grey 
interphase can be seen at the interfaces, along with morphological changes in the lithium electrodes. (i) Magnified cross-section of the same interface as shown in (f) after one full cycle at $1 \mathrm{~mA} \mathrm{~cm}^{-2}$. The volume of voids at the interface has increased significantly (overlaid with red on the left half of the image), along with growth of the interphase (demarcated by the red dashed line in the left half of the image).

Operando electrochemical cycling of symmetric Li/LSPS/Li cells was carried out with tomography scans interspersed at 15 min time intervals during the application of current. Galvanostatic voltage curves of two different cells cycled at $4 \mathrm{~mA} \mathrm{~cm}^{-2}$ and $1 \mathrm{~mA} \mathrm{~cm} \mathrm{~cm}^{-2}$ are shown in Fig. 1b, c. The shapes of these voltage curves are comparable to those measured under similar electrochemical conditions in a larger ex situ SSB cell (Fig. S4b, c). During these two experiments, our aim was to pass relatively large amounts of charge in each direction to understand how this affects stability. In the first half cycle of both experiments, over $4 \mathrm{mAh} \mathrm{cm}^{-2}$ of charge was transferred, corresponding to a theoretical lithium thickness of $\sim 20 \mu \mathrm{m}$. Under these conditions, the cells experienced a rapid increase in voltage towards the end of each half cycle (except for the first-half cycle of the cell in Fig. 1c). Small shifts of $\sim 10 \mathrm{mV}$ were observed in the voltage curve during exposure to the X-ray beam, which we attribute to X-ray interactions with the sample generating photoelectrons that could contribute to the measured voltage.

Figure 1d-i show cross-sectional images of Li/LSPS/Li symmetric cells before and after plating and stripping at $4 \mathrm{~mA} \mathrm{~cm}^{-2}$ (Fig. 1d, g) and $1 \mathrm{~mA} \mathrm{~cm}^{-2}$ (Fig. 1e, h). The contrast in the images is generated based on differences in the X-ray attenuation coefficients of the materials ${ }^{41}$. In each image, contrast between the LSPS with higher average $Z$ (brighter regions in the image) and the lower- $Z$ lithium metal electrodes (darker regions in the image) is clearly seen. The steel current collectors are also visible with white contrast at the top and bottom of the images in Fig. 1d, g. For both experiments, the images after cycling (Fig. 1g, h) reveal changes in the morphology of the lithium electrodes as a consequence of plating and stripping, as well as the formation of an 
interphase visible as the intermediate-contrast regions during plating. This change in contrast associated with interphase formation is due to the reaction of LSPS with lithium ${ }^{33}$, resulting in incorporation of additional $\mathrm{Li}$ and lowering of the average atomic number.

Magnified views of the same portion of the Li/LSPS interface before and after one full cycle at $1 \mathrm{~mA} \mathrm{~cm}^{-2}$ are shown in Fig. 1f and 1i. Before cycling (Fig. 1f), the lithium and LSPS are in physical contact at most regions of the interface, despite the roughness and asperities present. After electrochemical cycling, there are distinct regions with darker contrast than lithium metal visible at the Li/SSE interface (Fig. 1i). The right half of Fig. 1i is an unmodified image, while the left half features these darker-contrast regions segmented for visual clarity. The dark regions are found at the interface and not in the bulk region of lithium, suggesting that they represent a loss of lithium metal at the interface due to void formation. Line scans of the image intensity at a different interface before and after stripping lithium demonstrate that the darker-contrast regions are formed during stripping and exhibit lower intensity than lithium metal (Fig. S5a, b). The measured intensity of these regions is similar to pores within the bulk of the LSPS pellet (Fig. S5c), which further indicates that they are voids. The formation of voids at the interface is consistent with electrochemical experiments that have investigated lithium plating and stripping at high current densities $^{38-40}$. Our observations here indicate that we were able to successfully resolve voids using operando X-ray tomography. We partially attribute this result to the use of a monochromatic source, which improves the sensitivity to X-ray absorption variations in the sample. 


\section{Interphase Formation at the Li/LSPS Interface}

Reconstructed images reveal that a large amount of interphase is formed at the cathodic interface (Fig. 2a). To determine the relative fractions of current contributing to interphase growth vs. plating, we quantified the volumes of the interphase and the lithium electrodes by segmenting these regions in a subvolume (see Methods). Figure $2 \mathrm{~b}$ shows the change in these volumes relative to their initial value. As expected, the lithium volume at the anodic interface decreases as lithium is removed and transported to the cathodic interface. However, the lithium volume at the cathodic interface (shown in Fig. 2a) does not increase with time, which should occur if lithium metal is being deposited. Simultaneously, the interphase volume at this interface increases substantially (Fig. 2b). Together, these observations show that the current at the cathodic interface results in the electrochemical growth of the interphase rather than the deposition of lithium, and that the lithium metal electrode at this interface is actually consumed (albeit to a lesser extent than the other lithium electrode being stripped) due to the continuous chemical reaction that also contributes to interphase formation. Figure $\mathrm{S} 6$ shows results for the experiment at $4 \mathrm{~mA} \mathrm{~cm}^{-2}$, indicating that cycling at a higher current density results in similar interphase growth and electrochemical processes.

These findings demonstrate that the vast majority of applied current is used to electrochemically form the interphase. Reduction at the Li/LSPS interface does not actually involve measurable lithium deposition, and lithium loss due to interphase formation is inevitable. This observation is important because although interphase formation is well documented ${ }^{16,19,20,52}$, the extent to which this process affects the electrochemistry in various materials is not well understood. Because no metal deposition is occurring, we use the terminology "reduction" instead of "plating" to describe this cathodic process throughout the remainder of this paper. 


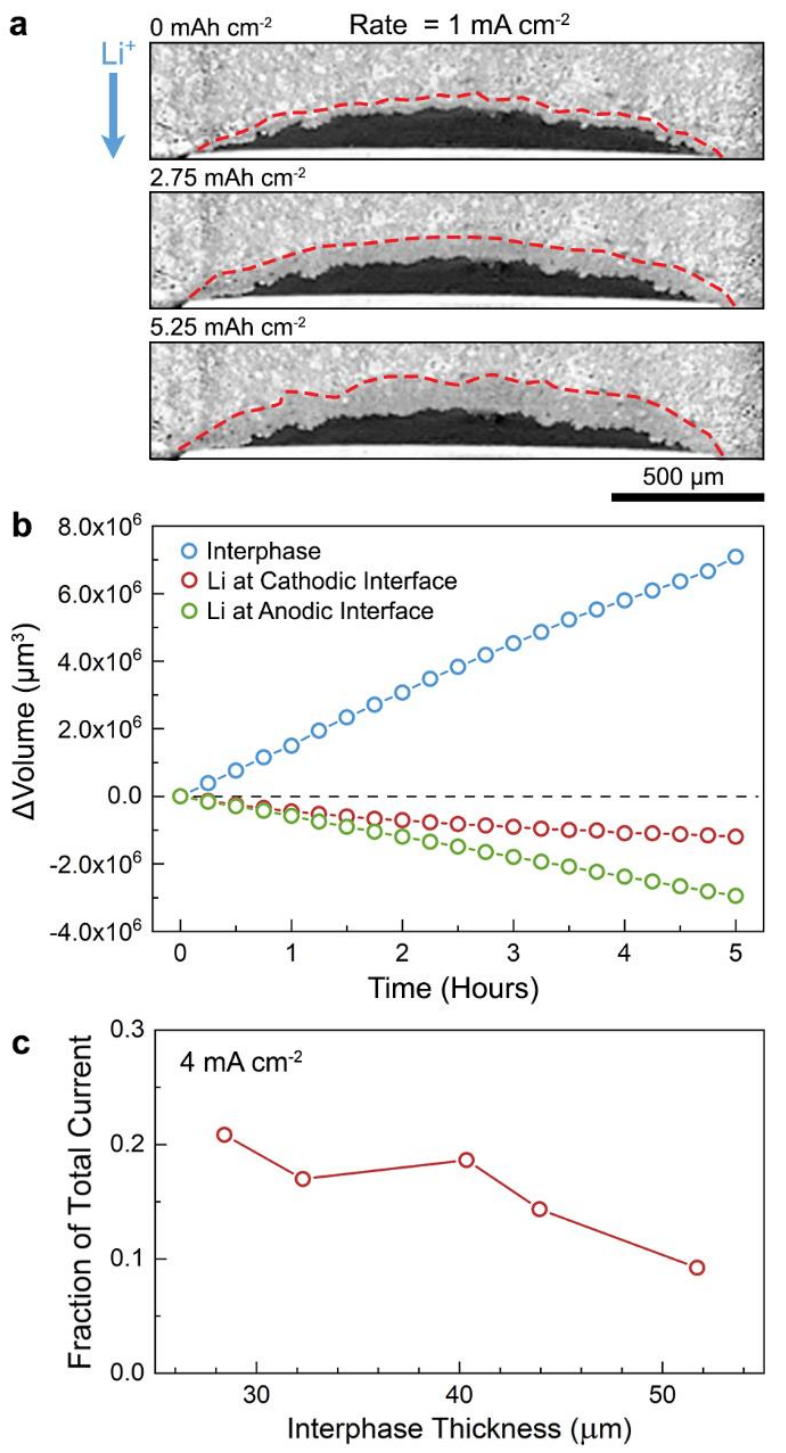

Figure 2. Analysis of interphase growth and electrochemical behavior. (a) Time lapse of an $\mathrm{Li} / \mathrm{LSPS}$ interface during reduction at $1 \mathrm{~mA} \mathrm{~cm}^{-2}$. The red dashed lines indicate the boundary of the interphase and pristine LSPS phase. (b) Plot showing the change in volume of the interphase at this interface, as well as both lithium electrodes, during the first half cycle at $1 \mathrm{~mA} \mathrm{~cm}^{-2} .340$ $\mu \mathrm{m}$ by $340 \mu \mathrm{m}$ by $340 \mu \mathrm{m}$ subvolumes were used for this analysis. (c) Fraction of total current due to oxidation of lithium metal, plotted as a function of the interphase thickness. This data were taken from an operando experiment with 30 minute cycles at a current density of $4 \mathrm{~mA} \mathrm{~cm}^{-2}$ (Fig. S7).

A consequence of the substantial interphase growth is that failure in Li/LSPS/Li cells does not occur due to lithium filament-induced short circuiting. No lithium filaments were observed to form in LSPS during our experiments, whereas X-ray imaging has previously shown the presence of lithium filaments in argyrodite SSEs after cycling ${ }^{48}$. Short circuiting was also never observed 
in our additional ex situ electrochemical measurements, similar to previous results with other SSEs that form a mixed-conducting interphase ${ }^{20,33,53}$. It is likely that all SSEs that form mixedconducting interphases will not experience substantial Li filament growth, with the caveat that there could be material-dependent balances between the rates of lithium deposition and interphase formation which may be altered at very high current densities.

Using the measured reduction in the volume of a lithium metal electrode during oxidation, we analyzed the fraction of the total applied current arising from lithium metal stripping. For the first and second half cycles of the experiment run at $1 \mathrm{~mA} \mathrm{~cm}^{-2}$ (Fig. 1c), these current fractions are 0.72 and 0.51 , respectively. Thus, a large portion of the current is being produced by interphase oxidation instead of lithium metal stripping. Reversible interphase redox activity has been shown in many SSEs ${ }^{24,52,54,55}$, and it can contribute a large amount of capacity during cycling. The decrease in this value in the second half-cycle indicates that stripping from a previously reduced interface alters the balance of electrochemical processes.

We further investigated this behavior using an operando experiment with a higher nominal current density $\left(4 \mathrm{~mA} \mathrm{~cm}^{-2}\right)$ and shorter cycle times to more steadily increase the total interphase thickness (Fig. S7). Figure 2c shows the fraction of current produced by stripping lithium metal at the top interface versus the thickness of the interphase at this interface when stripping begins. This plot demonstrates that less current arises directly from lithium stripping as the interphase thickness increases, suggesting that an increasing fraction of current comes from oxidation of lithium species in the interphase. We attribute this behavior to the increased total interphase volume present and the intimate contact at the interphase/LSPS interface. Finally, a greater fraction of current comes from oxidation of the interphase at higher currents, indicating that rate plays an important role as well. 


\section{Void Formation and Contact Loss at the Li/LSPS Interface}

Three different types of interfacial void evolution behavior were observed during cycling (Fig. 3). Each subfigure in Fig. 3 shows the exact same location at the lithium/SSE interface before and after an electrochemical process, with the voids at the interface automatically segmented (see Methods) and overlaid in red. Figure S8 contains the images without red overlays. The most common process is the formation of voids during stripping at an interface (Fig. 3a). The creation of void volume during stripping is due to the removal of $\mathrm{Li}^{+}$ions faster than the lithium metal can be replenished either through self-diffusion or mechanical deformation. Our observation of void formation at the anodic interface is consistent with recent studies that have demonstrated polarization at the stripping electrode ${ }^{38-40}$. Because the Li/LSPS interfaces in our study exhibited local nonuniformity, we also analyzed the possible influence of interface morphology on void formation (Fig. S9). Notably, our analysis shows that the tendency to form voids during stripping is independent of the local roughness of the interface. The second phenomenon we observed is the closing of voids during reduction (Fig. 3b). Despite there being no measurable lithium metal deposition that would fill voids, we suspect that local compression due to volume expansion and growth of the interphase can close some voids. However, this process was not observed in every experiment, suggesting that interphase formation is generally not effective at closing voids. The third phenomenon observed is the formation of voids at the cathodic interface (Fig. 3c). This behavior is counterintuitive, as mass is being transported to and added at this interface. However, the lithium electrode is being consumed due to a chemical side reaction (previously shown in Fig. 2b), which likely results in the void formation seen in Fig. 3c. 


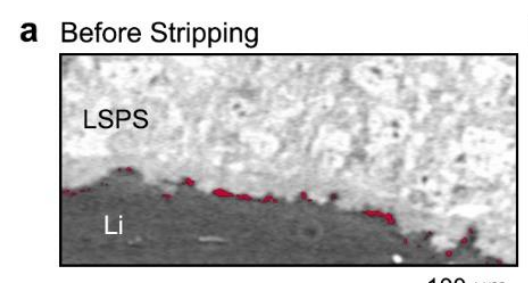

b Before Reduction

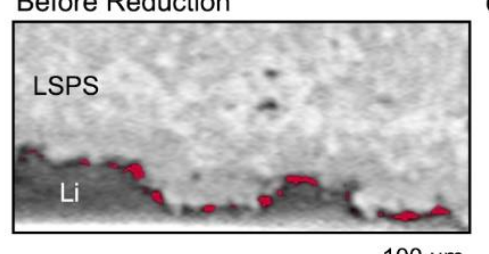

c Before Reduction
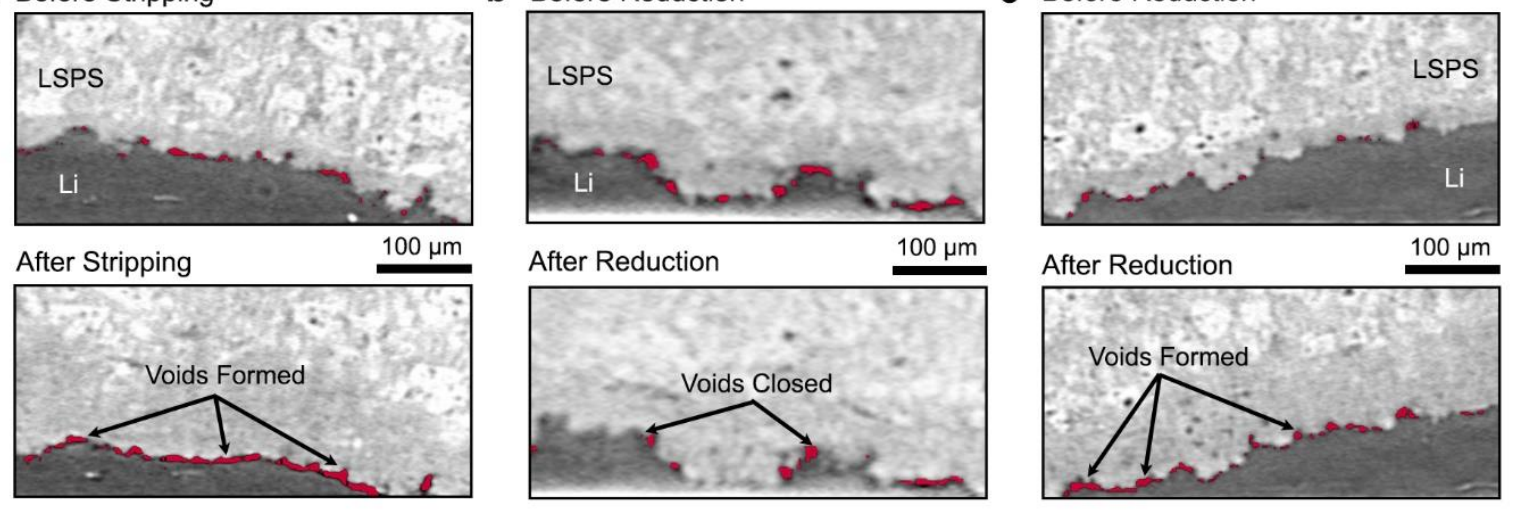

Figure 3. Evolution of voids at the Li/LSPS interface. (a) Cross-sectional images of the Li/LSPS interface before (top) and after (bottom) stripping at $1 \mathrm{~mA} \mathrm{~cm}$. The red overlays in these images represent the pixels identified as voids during the segmentation process, which shows that void growth occurs during stripping. (b) Cross-sectional images of the Li/LSPS interface before and after reduction at $4 \mathrm{~mA} \mathrm{~cm}{ }^{-2}$ show that voids can be closed during reduction. (c) Cross-sectional images before and after reduction at $1 \mathrm{~mA} \mathrm{~cm}{ }^{-2}$ show that voids can also be formed during reduction.

The image contrast between voids and lithium enables real-time determination of how the contact area between lithium and LSPS changes at both interfaces and how it influences electrochemistry. Figure 4a shows the Li/LSPS contact areas at both the top and bottom interfaces for the cell cycled at $1 \mathrm{~mA} \mathrm{~cm}{ }^{-2}$. Stripping occurs at the top interface for the first $\sim 5 \mathrm{~h}$, after which the current is reversed and stripping begins at the bottom interface. The electrochemical signature in the first half-cycle (i.e., during stripping at the top interface) only shows a minor increase in overpotential. The low overpotential is reflected in the contact areas at both interfaces, which do not change significantly in the first half cycle. After reversing the current, the cell voltage begins to increase in magnitude at a slow rate. However, after cycling for $\sim 9$ hours the cell rapidly polarizes to $-4 \mathrm{~V}$. During this time, the interfacial contact area at the bottom interface decreases by $52 \%$, mirroring the increasing overpotential. We note that in this two-electrode experiment, the measured voltage includes contributions from impedance at both interfaces. 
The spatiotemporal evolution of contact at each interface is visualized in Fig. 4b, c. These images show the projected contact area at different times during cycling. At time $=0 \mathrm{~h}$, there are already numerous regions without interfacial contact that are likely a result of insufficient stack pressure $^{48}$, as well as the non-planar interfaces created in this study. This is an important result, as it means the effective current density when cycling begins is actually 4-5 times higher than the nominal value. The projections for the top interface (Fig. 4b) show that there is only minor contact loss throughout the entire experiment, and contact actually increases slightly during stripping. Figure S10 shows that the physical locations at which contact is present at the top interface also shift during this half cycle, despite the small changes in total contact area. This result further emphasizes the dynamic nature of these interfaces. The contact projections of the bottom interface (Fig. 4c) show a more substantial change in contact area. A contact loss of $-8.9 \%$ can be seen over the first $4 \mathrm{~h}$ (between projections (i) and (ii)), despite no stripping at the bottom interface occurring. This evolution can be attributed to the formation of voids during reduction (Fig. 3c). However, these changes are not large enough to significantly polarize the cell until $\sim 9$ hours of cycling. A dramatic contact loss of $-47.1 \%$ at the bottom interface occurs between projections (iii) and (iv) during stripping of this interface, which corresponds to the period of rapid cell polarization. The contact loss at the bottom interface is greater in the final hour of cycling than the first 9.25 hours. 

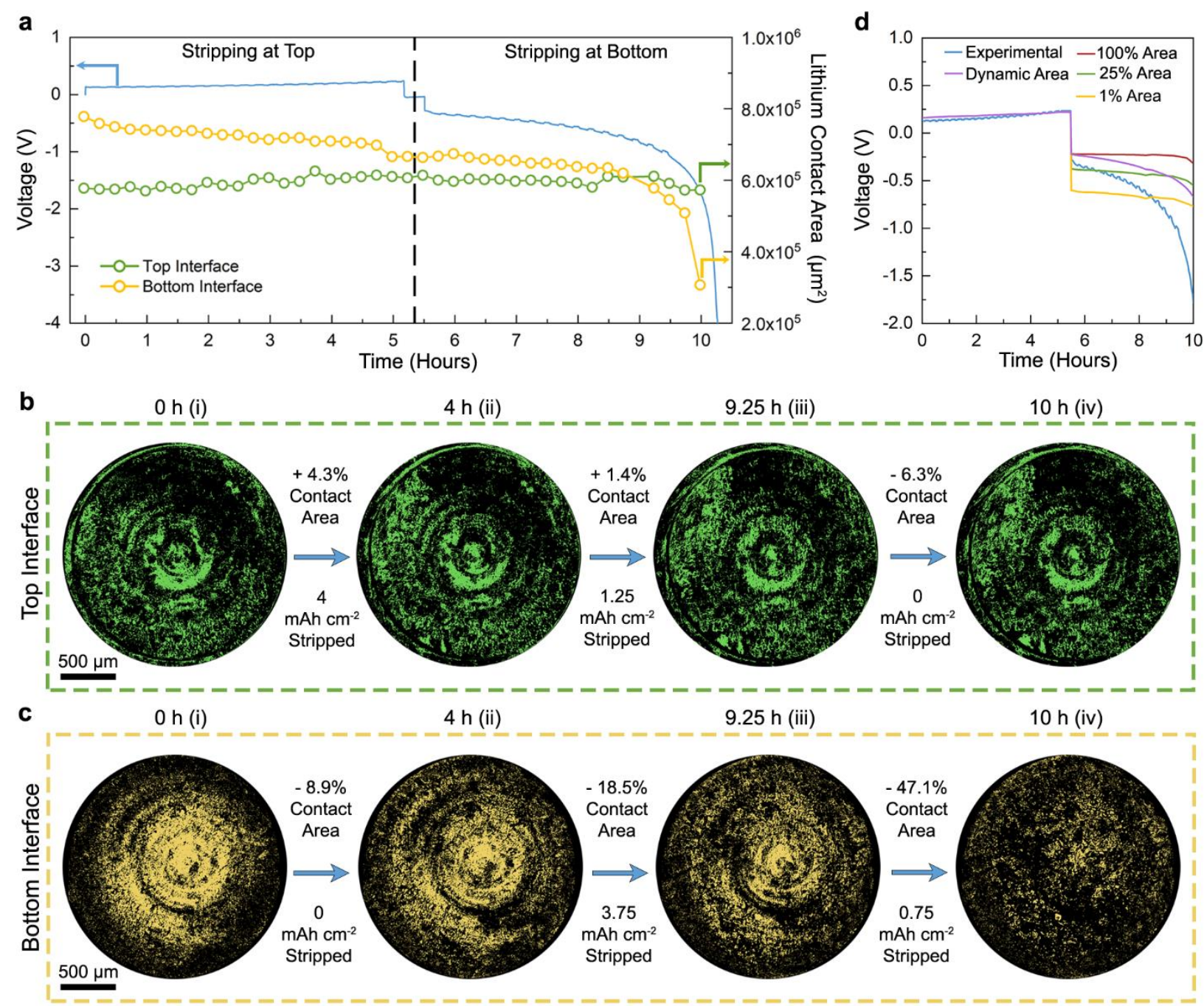

Figure 4. Relating interfacial contact area to cell electrochemistry. (a) Plot of the voltage curve measured during cycling at $1 \mathrm{~mA} \mathrm{~cm}^{-2}$ (blue line) and the corresponding lithium contact area of the top (green) and bottom (yellow) Li/LSPS interfaces in this cell. (b) Projections of the Li/LSPS contact area at the top interface. The percent change in contact area and lithium capacity removed between projections are indicated. (c) Projections of the bottom interfacial contact area. (d) Comparison between the experimental voltage curve (blue) and theoretical voltage curves using $100 \%$ of the experimental contact area in the second half cycle (red), 25\% (green), 1\% (yellow), and dynamically varied contact area (purple).

The correlation between interfacial contact and the increase in cell voltage suggests that the loss of contact at the anodic interface drives failure, despite the continuous growth of interphase during the experiments. Further analysis shows that in the regions of relatively low voltage $(<\sim 0.8$ V), the cell voltage is linearly related to the effective current density at the anodic interface (Fig. 
S11). At higher voltages, this dependence diverges from linearity, which indicates that factors such as differing redox processes or transport limitations in the interphase likely contribute at high overpotentials. To further investigate the effect of contact loss on cell failure, we implemented an electrochemical model with our cell geometry that incorporates Butler-Volmer kinetics at the $\mathrm{Li} / \mathrm{SSE}$ interface, as well as transport in the SSE and the growing interphase (see Methods). Figure $4 \mathrm{~d}$ shows the results for the situation in which the voltage of the first half cycle was matched to the experiment through choice of interphase conductivity. These conditions were then used to predict the voltage of the second half cycle based on the experimentally-measured contact area variation of the bottom interface (red curve). While there is an increase in overpotential as contact area decreases, the predicted cell voltage under these conditions is much lower than that which is measured. Reducing the contact area by fixed amounts in simulations of the second half cycle (green and yellow curves) increases the overpotential, but the slope of the curve still differs from experiment. Dynamically reducing the contact area during the second half cycle provides a qualitatively similar curve (purple curve), but matching to the experiment requires imposition of extremely low contact area (Fig. S12).

The mismatch between the predicted and experimental voltage curves suggests that the contact loss and associated increase in local current density likely drive other factors that cause the rapid overpotential increase which are not captured in this relatively simple model. For instance, the model assumes a continuous contact area, which is different than the distributed point contacts visible in Fig. 4c(iv). Point contacts can cause much higher local current densities (current constrictions) at the edges of the contacting regions ${ }^{56}$, which would exacerbate overpotential increases. Furthermore, our ability to quantify contact area is limited by the spatial resolution of these experiments, and some smaller voids that contribute are likely undetected. Lastly, redox of 
the interphase and mass transport limitations in this region would both be affected by increased local current density due to contact loss, but these are difficult to incorporate into this model due to experimental uncertainties. Thus, we conclude that the loss of contact at the stripping interface is the underlying phenomenon that sets into motion a variety of other possible contributing factors that cause cell failure in this system.

\section{Volumetric Changes and Partial Molar Volume Mismatch}

Our dataset also allows us to quantify the impact of volume changes at the electrodes and interphases on displacements at distant locations during cycling. Figure 5a shows a portion of a lithium electrode that undergoes stripping at $1 \mathrm{~mA} \mathrm{~cm}^{-2}$ during the first half cycle; this electrode decreases in volume during stripping, as detailed in Fig. 2. The Li/SSE interface moves upward $\sim 17 \mu \mathrm{m}$, as indicated by the red lines in Fig. $5 \mathrm{a}$, due to the lithium electrode receding during stripping and the simultaneous expansion of the interphase at the opposite interface (not shown). Through this process, however, the current collector at this interface moves downward by $\sim 20 \mu \mathrm{m}$ (blue shaded lines in Fig. 5a). The magnitude of these shifts (on the order of tens of micrometers) is significant because voids have an average thickness of $\sim 5 \mu \mathrm{m}$.

The LSPS pellet also undergoes nonuniform physical displacements during cycling. The vector field map in Fig. 5b shows the displacement of different positions within the SSE over the second half cycle, during which reduction (i.e., interphase growth) is happening at the top interface. Each vector in Fig. $5 \mathrm{~b}$ is scaled by five times the observed displacement of the point at the base of the vector. The maximum displacement measured in the SSE is $\sim 20 \mu \mathrm{m}$, which is consistent with the upward movement of the Li/LSPS interface in the previous half-cycle (Fig. 5a). These displacements show that the LSPS material moves nonuniformly towards the anodic interface, with greater displacements near the center of the pellet compared to the edges of the 
pellet. The different displacements at the center and the edges of the pellet will result in shear strain and nonuniform stresses, which could accelerate mechanical damage.
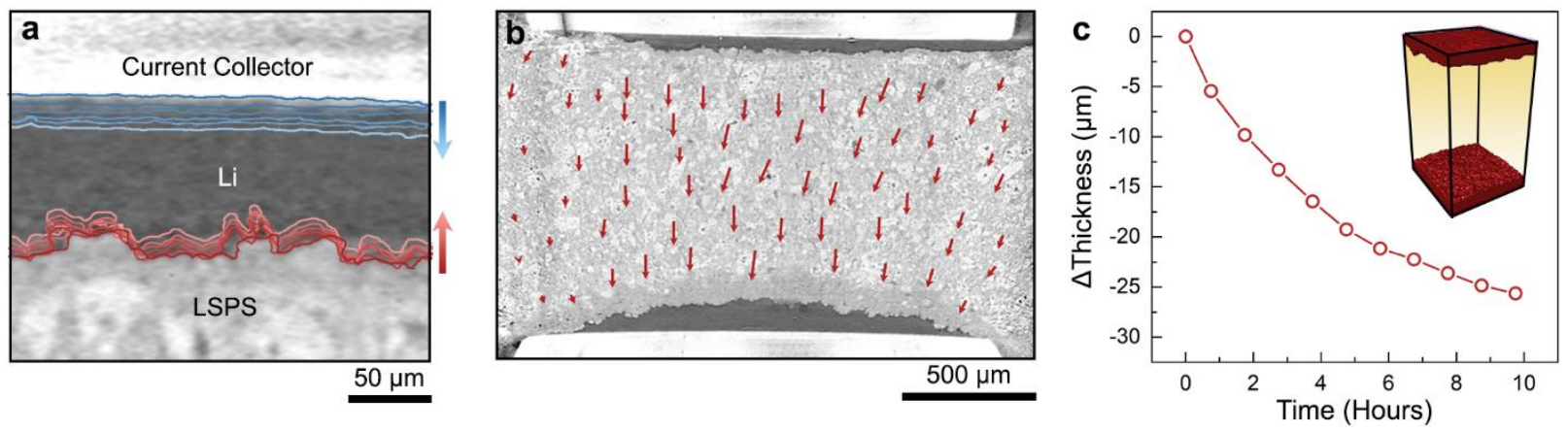

Figure 5. Displacements and volume changes within a cell. (a) The position of the Li/LSPS interface and the current collector tracked during stripping of the lithium. The arrows indicate the direction of motion of each interface; darker lines represent positions at earlier times during stripping, with the transition to lighter lines as stripping proceeds. (b) Vector map showing physical displacements of the point at the base of each vector as lithium is stripped from the bottom interface at a current density of $1 \mathrm{~mA} \mathrm{~cm}{ }^{-2}$. The magnitude of each vector is scaled by a factor of five for visual clarity. (c) Change in the thickness of the Li/LSPS/Li stack subvolume (visualized in the inset) during cycling at $1 \mathrm{~mA} \mathrm{~cm} \mathrm{~cm}^{-2}$.

The displacement of the LSPS pellet, coupled with the observation of current collector displacement, indicates a decrease in overall volume of the Li/LSPS/Li stack during cycling. Volume changes of the stack were analyzed in greater detail by tracking a $680 \mu \mathrm{m}$ by $680 \mu \mathrm{m}$ by $1360 \mu \mathrm{m}$ subvolume that encompasses the entire thickness of the stack between the current collectors. A segmented 3D visualization of this subvolume is shown in Fig. 5c, along with a plot of the change in thickness over time. The total thickness, and therefore volume, of the symmetric cell stack (i.e., all materials between the current collectors) decreases during cycling. Since the overall electrochemical process involves stripping lithium at one interface to form interphase at the other, this net volume loss can be explained if the partial molar volume of $\mathrm{Li}$ in the interphase is less than the partial molar volume of $\mathrm{Li}$ in lithium metal. Indeed, the partial molar volume of $\mathrm{Li}$ in most battery cathode and lithium binary compounds is less than that in lithium metal, and the compounds that form during interphase formation are expected to be similar ${ }^{32,57}$. As a result of this 
partial molar volume mismatch, the LSPS pellet and current collectors are displaced during cycling under stack pressure and there is a net decrease in cell volume due to continuous interphase formation. The different magnitudes of the displacements at different positions within the LSPS occur because the lithium electrodes at the edges of the cell are thinner, resulting in less interphase growth and volume change at these locations. Electrochemical cycling of an ex situ Li/LSPS/Li cell outfitted with a force sensor (Fig. S13) shows that the stack pressure decreases during cycling, which we suspect is due to the volume loss within the cell. Stack pressure plays a critical role in maintaining interfacial contact, and increasing the stack pressure of the operando tomography cell was found to increase the contact area while simultaneously decreasing cell voltage (Fig. S14). Thus, we conclude that the partial molar volume mismatch and volume loss in our cells leads to decreased stack pressure, which could exacerbate overpotential increases and is generally detrimental for the stability of SSBs.

Our observation of cell volume shrinkage is different than expected for $\mathrm{Li} / \mathrm{SSE} / \mathrm{Li}$ symmetric cells that undergo only lithium plating and stripping, as there would be no partial molar volume mismatch between the electrodes. Importantly, however, our results are relevant for designing full cells that contain a lithium metal anode and an intercalation or conversion cathode. As previously mentioned, Li has lower partial molar volume within most cathode materials than in lithium metal ${ }^{32}$, which is akin to the interphase found in our system. In both cases, then, the volume lost at the stripped lithium metal interface is greater than the volume gained when lithium is added to the cathode or interphase due to the lower partial molar volume of these phases, resulting in an overall reduction in cell volume. Our results highlight the challenges that partial molar volume mismatches present in creating full cells with controlled stack pressure throughout cycling $^{31,32,58}$, and they also indicate that studying Li/SSE/Li symmetric cells in which lithium 
metal deposition dominates may not be entirely representative of the chemo-mechanical conditions present in full cells.

\section{$\underline{\text { Conclusion }}$}

The chemo-mechanical phenomena we have observed herein are expected to play important roles during operation of SSBs based on a wide variety of SSE chemistries beyond LSPS. Contact evolution and volumetric changes are inherent challenges in SSBs, and nearly all SSEs decompose to some extent to form an interphase. Our results, along with continued use of operando X-ray tomography and other such techniques, will help guide the development of SSBs with high energy density and long lifetime. In particular, the dynamics of lithium filament growth and the effects of incorporating different composite cathodes are key scientific aspects that require greater understanding to successfully integrate lithium metal within SSBs. We have shown that it is possible to conduct operando imaging of contact loss at Li/SSE interfaces, which could help elucidate how lithium filaments nucleate and grow during charge. It is also important to study how volume change mismatches between electrodes in full cell architectures can alter mechanical stress

throughout the cell ${ }^{31,32}$. The displacement of components, localized stress variations, and loss of pressure caused by introducing a cathode could have an impact on contact at the Li/SSE interface, and thus the electrochemical performance of full SSBs. 


\section{$\underline{\text { Methods }}$}

\section{Cell Assembly}

$\mathrm{Li}_{10} \mathrm{SnP}_{2} \mathrm{~S}_{12}$ (LSPS) was purchased from NEI Corporation. A custom X-ray tomography cell was built for the operando synchrotron X-ray tomography experiments. The cell body was constructed out of PEEK to minimize X-ray attenuation and prevent short circuiting between the electrodes. The inner diameter was designed to be $2 \mathrm{~mm}$ to minimize the sample size, ensuring sufficient transmission to image the cell. Symmetric Li/LSPS/Li cells were assembled inside of an Ar-filled glovebox. $7 \mathrm{mg}$ of LSPS was loaded into the cell and pressed at a pressure of $225 \mathrm{MPa}$ to form a pellet approximately $1 \mathrm{~mm}$ thick. Lithium metal foils were punched out and attached to steel rods, which were then inserted into each side of the cell. To establish the pressure necessary to cycle these cells at high current densities, screws were used to compress the rods against the faces of the pellet. O-rings attached to the screw heads were simultaneously compressed to form a seal when the screw was inserted. The torque applied to the screws was $0.25 \mathrm{~N}-\mathrm{m}$ (estimated to be greater than $10 \mathrm{MPa}$ ) for each experiment unless otherwise specified. Graphite foils were placed between the steel rods and screws to prevent the rods from rotating and damaging the Li/SSE interface. All operando electrochemical measurements were performed using a Bio-Logic SP-150 potentiostat.

\section{Synchrotron X-Ray Computed Microtomography Experiments}

Operando X-ray computed microtomography experiments were conducted at the Advanced Photon Source's 2-BM beamline. Monochromatic X-rays with an energy of $28 \mathrm{keV}$ were chosen to maximize transmission in our samples based on attenuation length calculations (Fig. S3). 1500 projections were taken with an exposure time of $210 \mathrm{~ms}$ while rotating the sample $180^{\circ}$. An Oryx 5.0 MP Mono 10GigE detector and a 2x magnification lens were used for the optics 
in this setup. The sample to detector distance for all experiments was $100 \mathrm{~mm}$. We were able to achieve a voxel size of $1.7 \mu \mathrm{m}$ while fitting the entire sample within the field of view of $\sim 4.2 \mathrm{x}$ $1.4 \mathrm{~mm}^{2}$. The time required to complete a scan under these conditions was approximately $7 \mathrm{~min}$. The raw data was reconstructed with TomoPy using the Gridrec method ${ }^{59}$.

\section{Segmentation Analysis}

The reconstructed images were segmented using MATLAB to identify the lithium volume, interfacial contact area, and void volume. A dynamic cropping procedure was applied to select the appropriate regions of interest. To identify lithium metal, the cropped images were analyzed column by column from top to bottom. Differences in intensity between the steel rod and lithium electrode were used to define the upper boundary. The algorithm continued to recognize pixels as lithium as long as their intensity was below the specified threshold. The identification of lithium stopped when the average intensity of the next 3-5 pixels was above a value typical of the interphase.

The same algorithm was used to segment the contact areas with one modification. After identifying lithium in every column, the contact algorithm evaluated the bottom 5 pixels in search for values below the void intensity threshold. If pixels with these intensities were found, then the entire column was deleted. The segmented slices were projected onto the $\mathrm{z}$ axis such that columns with lithium and no voids would count as a white pixel, columns without lithium would be a black pixel, and columns with lithium and voids at the bottom of the lithium would be a black pixel. This algorithm was used to produce the images in Fig 4.

Void segmentation was done with an independent algorithm. In each column, the algorithm went from the bulk of the LSPS pellet identifying pixels with intensities below the void intensity threshold. Once such a pixel was found, it would continue identifying the pixels above it with 
intensities below the void threshold. If the average intensity of the next 3-5 pixels was above the void threshold and below the lithium threshold, all of the identified pixels were segmented as an interfacial void. If the average intensity above the void was higher than the lithium threshold (indicating that this void was a pore in the bulk), it was discarded and the algorithm would continue to sweep the column upwards.

The interphase volumes were segmented using the WEKA trainable segmentation package built into FIJI/ImageJ. This analysis was conducted using $340 \mu \mathrm{m}$ by $340 \mu \mathrm{m}$ by $340 \mu \mathrm{m}$ subvolumes. Classifiers for different phases in the sample were created and then manually identified over many cross-sections. These inputs were then used to train a model that could identify the classified phases over the entire subvolume. The resulting RGB images were then binarized to isolate the interphase class and processed using MATLAB.

Each segmented slice for lithium volume, void volume, interphase volume, and contact area was saved having the positively identified elements as white pixels and the negatives as black pixels. The volumes were calculated by adding up the number of white pixels through all the slices in a scan and using the voxel size $(1.7 \mu \mathrm{m})$. Similarly, the contact area was calculated by adding the white pixels in the z-projections.

\section{Electrochemical Model}

To understand the mechanisms underlying the dynamic change in the cell voltage, an electrochemical model for a symmetric cell configuration was developed, which takes into account the contact areas at the top and bottom interfaces, growth of the interphase, and the electrolyte conductivity.

The kinetic overpotential at the top and bottom interfaces were calculated using the ButlerVolmer expression. 


$$
i_{B V}=i_{0}\left(\exp \left(\frac{\alpha_{a} F}{R T} \eta\right)-\exp \left(-\frac{\alpha_{c} F}{R T} \eta\right)\right)
$$

Here, $i_{B V}$ refers to the reaction current density, which was evaluated with respect to the experimentally extracted contact areas (distinct for the top and bottom interfaces). $i_{0}, \alpha_{a}, \alpha_{c}$, and $\eta$ refer to the exchange current density, cathodic and anodic charge transfer coefficients, and kinetic overpotential, respectively.

The potential drops across the electrolyte and interphase were calculated as follows:

$$
\Delta \phi_{j}=\frac{I_{a p p} l_{j}}{k_{j}}
$$

$I_{a p p}, k_{j}$, and $l_{j}$ correspond to the applied current density, ionic conductivity (electrolyte or interphase), and thickness (electrolyte or interphase), respectively. It is to be noted that the interphase thickness evolves over time and this input is derived from the experimental data. The cell voltage was computed by summing up contributions from the ohmic and kinetic potential drops, which have been described above. Owing to the high electronic conductivity of lithium metal, the ohmic drop across the metal electrode has been neglected in the above analysis.

\section{Acknowledgements}

This work is partially supported by the National Science Foundation under Award No. DMR1652471. M.T.M. acknowledges support from a Sloan Research Fellowship in Chemistry from the Alfred P. Sloan Foundation. J.A.L. acknowledges support from a NASA Space Technology Research Fellowship. F.J.Q.C. acknowledges support from Colciencias-Fulbright scholarship program cohort 2016. C.L. and H.L. acknowledge support from the Ministry of Trade, Industry \& Energy/Korea Institute of Energy Technology Evaluation and Planning (MOTIE/KETEP) (20194010000100). A portion of this work is supported by the Air Force Office of Scientific Research (AFOSR) under Grant FA9550-17-1-0130. This research used resources of the 
Advanced Photon Source, a U.S. Department of Energy (DOE) Office of Science User Facility operated for the DOE Office of Science by Argonne National Laboratory under Contract No. DEAC02-06CH11357.

\section{Author Contributions}

M. T. M., J. A. L., and F. J. Q. C. conceived the study. J. A. L., F. J. Q. C., Y. L., and T. S. M. conducted the operando X-ray tomography experiments. J. A. L., J. C. M., and J. T. designed and tested the cell housings. J. A. L. and D. P. conducted preliminary ex situ cycling experiments. S. Y. H. and C. L. developed pressure measurements for cell housings. F. D. C. and P. S. assisted with the use of beamline 2-BM and reconstruction processing. A. V., B. S. V., and P. P. M. developed the electrochemical model. J. A. L. and F. J. Q. C. segmented and analyzed the data, and also wrote the manuscript with M. T. M.

\section{Competing Interests}

The authors declare no competing interests.

\section{Data Availability}

The data that support the plots within this paper and other findings of this study are available from the corresponding author upon reasonable request.

\section{Additional Information}

Correspondence and requests for materials should be addressed to M.T.M. 


\section{References}

1. Manthiram, A., Yu, X. \& Wang, S. Lithium battery chemistries enabled by solid-state electrolytes. Nat. Rev. Mater. 2, 16103 (2017).

2. Famprikis, T., Canepa, P., Dawson, J. A., Islam, M. S. \& Masquelier, C. Fundamentals of inorganic solid-state electrolytes for batteries. Nat. Mater. 18, 1278-1291 (2019).

3. Tan, D. H. S., Banerjee, A., Chen, Z. \& Meng, Y. S. From nanoscale interface characterization to sustainable energy storage using all-solid-state batteries. Nat. Nanotechnol. 15, 170-180 (2020).

4. Janek, J. \& Zeier, W. G. A solid future for battery development. Nat. Energy 1, 16141 (2016).

5. Murugan, R., Thangadurai, V. \& Weppner, W. Fast lithium ion conduction in garnet-type $\mathrm{Li}_{7} \mathrm{La}_{3} \mathrm{Zr}_{2} \mathrm{O}_{12}$. Angew. Chem. Int. Ed. 46, 7778-7781 (2007).

6. Kamaya, N. et al. A lithium superionic conductor. Nat. Mater. 10, 682-686 (2011).

7. Deiseroth, H. J. et al. $\mathrm{Li}_{6} \mathrm{PS}_{5} \mathrm{X}$ : A class of crystalline Li-rich solids with an unusually high Li+ mobility. Angew. Chem. Int. Ed. 47, 755-758 (2008).

8. Thokchom, J. S. \& Kumar, B. Composite effect in superionically conducting lithium aluminium germanium phosphate based glass-ceramic. J. Power Sources 185, 480-485 (2008).

9. Mizuno, F., Hayashi, A., Tadanaga, K. \& Tatsumisago, M. High lithium ion conducting glass-ceramics in the system $\mathrm{Li}_{2} \mathrm{~S}_{-} \mathrm{P}_{2} \mathrm{~S}_{5}$. Solid State Ion. 177, 2721-2725 (2006).

10. Zhou, L., Assoud, A., Zhang, Q., Wu, X. \& Nazar, L. F. New family of argyrodite thioantimonate lithium superionic Conductors. J. Am. Chem. Soc. 141, 19002-19013 (2019).

11. Kato, Y. et al. High-power all-solid-state batteries using sulfide superionic conductors. Nat. Energy 1, 16030 (2016).

12. Lewis, J. A., Tippens, J., Cortes, F. J. Q. \& McDowell, M. T. Chemo-mechanical challenges in solid-state batteries. Trends Chem. 1, 845-857 (2019).

13. Nolan, A. M., Zhu, Y., He, X., Bai, Q. \& Mo, Y. Computation-accelerated design of materials and interfaces for all-solid-state lithium-ion batteries. Joule 2, 2016-2046 (2018).

14. Xiao, Y. et al. Understanding interface stability in solid-state batteries. Nature Rev. Mater. 5, 105-126 (2020).

15. Richards, W. D., Miara, L. J., Wang, Y., Kim, J. C. \& Ceder, G. Interface stability in solidstate batteries. Chem. Mater. 28, 266-273 (2016).

16. Zhu, Y., He, X. \& Mo, Y. Origin of outstanding stability in the lithium solid electrolyte materials: Insights from thermodynamic analyses based on first-principles calculations. ACS Appl. Mater. Interfaces 7, 23685-23693 (2015).

17. Ong, S. P. et al. Phase stability, electrochemical stability and ionic conductivity of the $\mathrm{Li}_{10 \pm 1} \mathrm{MP}_{2} \mathrm{X}_{12}(\mathrm{M}=\mathrm{Ge}, \mathrm{Si}, \mathrm{Sn}, \mathrm{Al}$ or $\mathrm{P}$, and $\mathrm{X}=\mathrm{O}, \mathrm{S}$ or $\mathrm{Se})$ family of superionic conductors. Energy Environ. Sci. 6, 148-156 (2013).

18. Hartmann, P. et al. Degradation of NASICON-type materials in contact with lithium metal: Formation of mixed conducting interphases (MCI) on solid electrolytes. J. Phys. Chem. C 117, 21064-21074 (2013). 
19. Wenzel, S. et al. Direct observation of the interfacial instability of the fast ionic conductor $\mathrm{Li}_{10} \mathrm{GeP}_{2} \mathrm{~S}_{12}$ at the lithium metal anode. Chem. Mater. 28, 2400-2407 (2016).

20. Lewis, J. A. et al. Interphase morphology between a solid-state electrolyte and lithium controls cell failure. ACS Energy Lett. 4, 591-599 (2019).

21. Ma, C. et al. Interfacial stability of Li metal-solid electrolyte elucidated via in situ electron microscopy. Nano Lett. 16, 7030-7036 (2016).

22. Wenzel, S., Sedlmaier, S. J., Dietrich, C., Zeier, W. G. \& Janek, J. Interfacial reactivity and interphase growth of argyrodite solid electrolytes at lithium metal electrodes. Solid State Ion. 318, 102-112 (2018).

23. Wenzel, S. et al. Interphase formation and degradation of charge transfer kinetics between a lithium metal anode and highly crystalline $\mathrm{Li}_{7} \mathrm{P}_{3} \mathrm{~S}_{11}$ solid electrolyte. Solid State Ion. 286, 24-33 (2016).

24. Schwietert, T. K. et al. Clarifying the relationship between redox activity and electrochemical stability in solid electrolytes. Nature Mater. 19, 428-435 (2020).

25. Cheng, E. J., Sharafi, A. \& Sakamoto, J. Intergranular Li metal propagation through polycrystalline $\mathrm{Li}_{6.25} \mathrm{Al}_{0.25} \mathrm{La}_{3} \mathrm{Zr}_{2} \mathrm{O}_{12}$ ceramic electrolyte. Electrochim. Acta 223, 85-91 (2017).

26. Porz, L. et al. Mechanism of lithium metal penetration through inorganic solid electrolytes. Adv. Energy Mater. 7, 1701003 (2017).

27. Han, F. et al. High electronic conductivity as the origin of lithium dendrite formation within solid electrolytes. Nat. Energy 4, 187-196 (2019).

28. Kazyak, E. et al. Li penetration in ceramic solid electrolytes: Operando microscopy analysis of morphology, propagation, and reversibility. Matter 2, 1025-1048 (2020).

29. Randau, S. et al. Benchmarking the performance of all-solid-state lithium batteries. Nat. Energy 5, 259-270 (2020).

30. Koerver, R. et al. Capacity fade in solid-state batteries: Interphase formation and chemomechanical processes in nickel-rich layered oxide cathodes and lithium thiophosphate solid electrolytes. Chem. Mater. 29, 5574-5582 (2017).

31. Zhang, W. et al. (Electro)chemical expansion during cycling: Monitoring the pressure changes in operating solid-state lithium batteries. J. Mater. Chem. A 5, 9929-9936 (2017).

32. Koerver, R. et al. Chemo-mechanical expansion of lithium electrode materials-on the route to mechanically optimized all-solid-state batteries. Energy Environ. Sci. 11, 2142-2158 (2018).

33. Tippens, J. et al. Visualizing chemomechanical degradation of a solid-state battery electrolyte. ACS Energy Lett. 4, 1475-1483 (2019).

34. Sharafi, A. et al. Surface chemistry mechanism of ultra-low interfacial resistance in the solid-state electrolyte $\mathrm{Li}_{7} \mathrm{La}_{3} \mathrm{Zr}_{2} \mathrm{O}_{12}$. Chem. Mater. 29, 7961-7968 (2017).

35. Krauskopf, T., Hartmann, H., Zeier, W. G. \& Janek, J. Toward a fundamental understanding of the lithium metal anode in solid-state batteries - An electrochemo-mechanical study on the garnet-type solid electrolyte $\mathrm{Li}_{6.25} \mathrm{Al}_{0.25} \mathrm{La}_{3} \mathrm{Zr}_{2} \mathrm{O}_{12}$. ACS Appl. Mater. Interfaces 11, 14463-14477 (2019).

36. Han, X. et al. Negating interfacial impedance in garnet-based solid-state Li metal batteries. Nat. Mater. 1, 572-579 (2017). 
37. Lee, Y. G. et al. High-energy long-cycling all-solid-state lithium metal batteries enabled by silver-carbon composite anodes. Nat. Energy 5, 299-308 (2020).

38. Kasemchainan, J. et al. Critical stripping current leads to dendrite formation on plating in lithium anode solid electrolyte cells. Nat. Mater. 18, 1105-1111 (2019).

39. Wang, M. J., Choudhury, R. \& Sakamoto, J. Characterizing the Li-solid-electrolyte interface dynamics as a function of stack pressure and current density. Joule 3, 2165-2178 (2019).

40. Koshikawa, H. et al. Dynamic changes in charge-transfer resistance at $\mathrm{Li}$ metal/ $/ \mathrm{Li}_{7} \mathrm{La}_{3} \mathrm{Zr}_{2} \mathrm{O}_{12}$ interfaces during electrochemical $\mathrm{Li}$ dissolution/deposition cycles. $J$. Power Sources 376, 147-151 (2018).

41. Pietsch, P. \& Wood, V. X-ray tomography for lithium ion battery research: A practical guide. Annu. Rev. Mater. Res. 47, 451-479 (2017).

42. Lin, F. et al. Synchrotron X-ray analytical techniques for studying materials electrochemistry in rechargeable batteries. Chem. Rev. 117, 13123-13186 (2017).

43. Shen, F., Dixit, M. B., Xiao, X. \& Hatzell, K. B. Effect of pore connectivity on Li dendrite propagation within LLZO electrolytes observed with synchrotron X-ray tomography. ACS Energy Lett. 3, 1056-1061 (2018).

44. Dixit, M. B. et al. Nanoscale mapping of extrinsic interfaces in hybrid solid electrolytes. Joule 4, 207-221 (2020).

45. Wu, X. et al. Operando visualization of morphological dynamics in all-solid-state batteries. Adv. Energy Mater. 9, 1901547 (2019).

46. Jolly, D. S. et al. Sodium/Na $\beta^{\prime \prime}$ alumina interface: Effect of pressure on voids. ACS Appl. Mater. Interfaces 12, 678-685 (2019).

47. Neumann, A. et al. Analysis of interfacial effects in all-solid-state batteries with thiophosphate solid electrolytes. ACS Appl. Mater. Interfaces 12, 9277-9291 (2020).

48. Doux, J. M. et al. Stack pressure considerations for room-temperature all-solid-state lithium metal batteries. Adv. Energy Mater. 10, 1903253 (2020).

49. Harry, K. J., Hallinan, D. T., Parkinson, D. Y., MacDowell, A. A. \& Balsara, N. P. Detection of subsurface structures underneath dendrites formed on cycled lithium metal electrodes. Nat. Mater. 13, 69-73 (2014).

50. Bron, P. et al. $\mathrm{Li}_{10} \mathrm{SnP}_{2} \mathrm{~S}_{12}$ : An affordable lithium superionic conductor. J. Am. Chem. Soc. 135, 15694-15697 (2013).

51. Zhang, W. et al. Interfacial processes and influence of composite cathode microstructure controlling the performance of all-solid-state lithium batteries. ACS Appl. Mater. Interfaces 9, 17835-17845 (2017).

52. Tarhouchi, I., Viallet, V., Vinatier, P. \& Ménétrier, M. Electrochemical characterization of $\mathrm{Li}_{10} \mathrm{SnP}_{2} \mathrm{~S}_{12}$ : An electrolyte or a negative electrode for solid state Li-ion batteries? Solid State Ion. 296, 18-25 (2016).

53. Cortes, F. J. Q., Lewis, J. A., Tippens, J., Marchese, T. S. \& McDowell, M. T. How metallic protection layers extend the lifetime of NASICON-based solid-state lithium batteries. $J$. Electrochem. Soc. 167, 050502 (2020).

54. Tan, D. H. S. et al. Elucidating reversible electrochemical redox of $\mathrm{Li}_{6} \mathrm{PS}_{5} \mathrm{Cl}$ solid electrolyte. ACS Energy Lett. 4, 2418-2427 (2019) 
55. Han, F., Gao, T., Zhu, Y., Gaskell, K. J. \& Wang, C. A battery made from a single material. Adv. Mater. 27, 3473-3483 (2015).

56. Greenwood, J. A. Constriction resistance and the real area of contact. Brit. J. Appl. Phys. 17 1621-1632 (1966).

57. Ahmad, Z. \& Viswanathan, V. Stability of electrodeposition at solid-solid interfaces and implications for metal anodes. Phys. Rev. Lett. 119, 056003 (2017).

58. Mistry, A. \& Mukherjee, P. P. Molar volume mismatch: A malefactor for irregular metallic electrodeposition with solid electrolytes. J. Electrochem. Soc. 167, 082510 (2020).

59. Gürsoy, D., de Carlo, F., Xiao, X. \& Jacobsen, C. TomoPy: A framework for the analysis of synchrotron tomographic data. J. Synchrotron Radiat. 21, 1188-1193 (2014). 